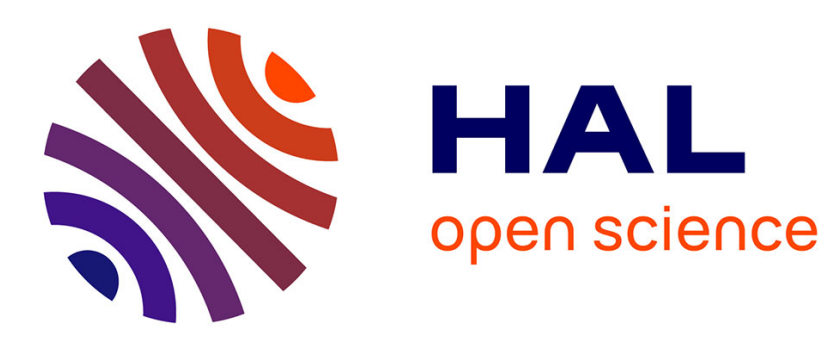

\title{
Les contiones militaires des colonnes trajane et aurélienne: les nécessités de l'adhésion
}

\author{
Jean-Michel David
}

\section{To cite this version:}

Jean-Michel David. Les contiones militaires des colonnes trajane et aurélienne: les nécessités de l'adhésion. John Scheid et Valérie Huet. Autour de la colonne aurélienne, geste et image sur la colonne de Marc Aurèle à Rome,, 108, Brepols, pp. 213-226., 2000, Bibliothèque de l'Ecole des hautes études, section des sciences religieuses. hal-01174153

\section{HAL Id: hal-01174153 https://hal.science/hal-01174153}

Submitted on 9 Jul 2015

HAL is a multi-disciplinary open access archive for the deposit and dissemination of scientific research documents, whether they are published or not. The documents may come from teaching and research institutions in France or abroad, or from public or private research centers.
L'archive ouverte pluridisciplinaire HAL, est destinée au dépôt et à la diffusion de documents scientifiques de niveau recherche, publiés ou non, émanant des établissements d'enseignement et de recherche français ou étrangers, des laboratoires publics ou privés. 
Les contiones militaires des colonnes Trajane et Aurélienne : les nécessités de l'adhésion

C'est généralement sous le nom d'adlocutio que les historiens de l'art romain désignent ces scènes qui, sur des monnaies ou d'autres monuments figurés célébrant la gloire impériale, représentaient un empereur debout, dressé sur un tribunal tenant un discours à ses soldats rangés eux-mêmes à ses pieds. De telles images contribuaient à la définition du pouvoir. C'est donc sans surprise qu'on les retrouve à plusieurs reprises sur les colonnes de $\operatorname{Trajan}^{1}$ et de Marc Aurèle.

Pour valoir un tel traitement, le moment était évidemment d'importance. L'empereur s'adressait à ses hommes. Ceux-ci lui répondaient par des acclamations. Ainsi se mettait en place un moment de communication qui avait pour effet de nouer (ou de renouer) des relations de confiance. L'interaction produisait en effet un rapport privilégié qui était luimême la garantie de l'autorité du chef et de l'obéissance de ses troupes. Un manque d'adhésion aurait en effet ouvert ou révélé une situation de crise dont la sanction aurait été soit la perte de pouvoir de l'un, soit la punition des autres.

En fait, le terme par lequel les sources littéraires le désignaient, était celui de contio ou

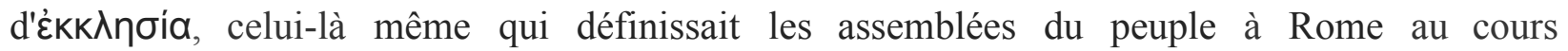
desquelles, anciennement, les magistrats présentaient les projets de loi ou les accusations contre ceux qu'ils poursuivaient et sur lesquels les comices devaient voter. Le mot se distinguait de celui d'adlocutio que l'on trouve sur les monnaies ${ }^{2}$ ou dans quelques inscriptions ${ }^{3}$ en ce sens que ce dernier ne désignait que le discours de l'empereur lui-même et non l'ensemble de la situation. Il marquait une continuité sur laquelle on reviendra car elle signifie en fin de compte que les soldats étaient compris sous ce terme comme des citoyens qui prenaient véritablement part à un rituel politique qui garantissait la légitimité du Prince ${ }^{4}$. Il

\footnotetext{
${ }^{1}$ Cf. K. LEHMANN-HARTLEBEN, Die Trajanssäule, ein römisches Kunstwerk zu Beginn der Spätantike, Berlin et Leipzig, 1926, pp. 11-24 ; M. WEGNER, Die kunstgeschichtliche Stellung des Marcussäule, J.d.I., 46, 1931, pp. 61-174, en part. pp. 104-115 ; P. G. HARNBERG, Studies in Roman Imperial Art, Uppsala, 1945, pp. 135-149 ; L. E. BAUMER, Adlocutio, Ikonographie und Prograrnmatik der kaiserlichen Heeresansprachen an der Trajanssäule, dans L. E. BAUMER, T. HÖLSCHER et L. W1NKLER, Narrative Systematik und politisches Konzept in den Reliefs der Traianssäule, Drei Fallstudien, J.d.I., 106, 1991, pp. 261-295. Cf. d'une façon plus générale, CICHORIUS, art. Ad locutio, R.E., 1, 1894.

${ }^{2}$ Cf. e.g., H. MATTINGLY \& E.A. SYDENHAM, The Roman Imperial Coinage, Londres, 1923, 1, p. 149, pl. X, 166.

${ }^{3}$ Cf. ILS, 9133, cf. $2487 ; 9133-9135$.

4 Je rejoins ici les analyses d'E. FLAIG, Den Kaiser herausfordern, Die Usurpation im römischen Reich, Francfort, 1992 (en part. pp. 159-164) qui a placé à juste titre la notion de consensus au cœur de la légitimité impériale. La même scène apparaît parfois comme désignant la fides exercitus (cf. e.g. H. MATTINGLY \& E.A. SYDENHAM, o.C., 1930, III, p. 378). Le terme met alors l'accent sur le rôle des soldats dans l'interaction.
} 
prenait en compte l'ensemble des [213] partenaires de la scène. C'est donc lui qu'il faut employer sauf à prendre le risque de concentrer sous celui d'adlocutio l'analyse sur la seule personne de 1 'orateur'.

La contio se définissait ainsi comme une situation de communication particulière qui avait ses règles de fonctionnement et répondait à un certain nombre de nécessités.

Or les sources qui nous la donnent à percevoir - et parmi elles évidemment, les représentations qui figurent sur les colonnes de Trajan et de Marc Aurèle - étaient elles aussi soumises à leurs propres contraintes sémantiques. Les contiones réelles, que l'on pourrait dire actuelles, se trouvaient en effet inscrites soit dans les narrations que les historiens anciens nous ont laissées, soit dans les scènes que les monuments figurés nous ont conservées. Les situations d'interaction qu'elles constituaient étaient ainsi incluses et utilisées dans d'autres modes de communication dont les fonctions étaient forcément différentes. L'image, par exemple, que Tacite ou l'auteur des représentations de la colonne Trajane donnaient de l'empereur, obéissait à d'autres nécessités, narratives ou iconographiques, que celles auxquelles celui-ci se soumettait lorsqu'il prenait la parole devant ses troupes. Le problème qui se pose alors est de déterminer avec le plus de précision possible la part qui revient à chacun de ces outils d'énonciation; en d'autres termes, de distinguer entre les différents cadres où la mise en œuvre de l'interaction se trouvait inscrite et qui lui imposaient les exigences de leurs propres langages. Non pas parce qu'en vertu d'on ne sait quel souci d'objectivité, il faudrait revenir des sources à une réalité de toute façon insaisissable, mais parce que les nécessités sémantiques à l'œuvre dans tous ces modes de communication, sont elles-mêmes révélatrices des rôles que jouaient les acteurs et des images que l'on donnait d'eux et aussi parce qu'il n'est guère possible de comprendre chacun d'eux autrement que par leurs relations réciproques. Ce n'est qu'à cette condition en tout cas que l'on pourra mieux comprendre le fonctionnement sémantique des colonnes de Trajan et de Marc Aurèle ${ }^{6}$.

Cette aporie de principe qui tient à ce que l'on ne sait strictement rien des contiones en dehors des récits ou des représentations qui en ont conservé le témoignage et que l'on ne peut pas en restituer le fonctionnement sans devoir s'affranchir des contraintes qui leur étaient propres, peut cependant être surmontée. On peut en effet chercher à identifier dans nos sources les conduites les plus récurrentes, celles dont on peut postuler qu'elles correspondaient aux comportements les plus nécessaires, à ceux qu'il était indispensable d'adopter. Il se trouve que ce sont les sources narratives qui fournissent le plus grand nombre de descriptions de contiones, qui indiquent les situations très diverses dans lesquelles elles se

\footnotetext{
${ }^{5}$ Tous les historiens de l'art désignent ces scènes sous ce nom. Ce n'est sans doute pas indifférent au fait qu'ils privilégient ainsi la vertu impériale.

${ }^{6}$ C'est ainsi que J. B. CAMPBELL, The Emperor and the Roman Army, 31 BC-AD 235, Oxford, 1984, pp. 69-88, mêle les diverses sources d'informations.
} 
tinrent et qui fournissent les renseignements les plus précis sur les circonstances dans lesquelles elles se déroulèrent, qu'elles fussent heureuses ou dramatiques, consensuelles ou conduites dans un état de crise. C'est donc de cette documentation qu'il faut partir pour restituer autant qu'on le peut les rituels qui marquaient la communication entre l'empereur et ses hommes. On pourra ensuite s'intéresser dans un deuxième temps aux nécessités sémantiques qui s'imposaient aux récits historiques et aux représentations figurées qui célébraient le pouvoir impérial sur les colonnes Trajane et Aurélienne. [214]

Le corpus des narrations de contiones au cours desquelles un chef s'adressait à ses hommes est considérable. Il comprend 138 cas pour la période royale et républicaine et 95 cas pour la période impériale qui ont été rassemblés dans un ouvrage récent par Monsieur Francisco Pina Polo ${ }^{7}$. La question se pose évidemment de la pertinence qu'il y aurait à employer des sources portant sur la période républicaine ou royale pour comprendre des monuments du deuxième siècle de notre ère. On doit cependant noter que lorsqu'il s'agissait d'événements très anciens, les historiens ne pouvaient reconstituer les comportements des chefs et de leurs hommes qu'à partir des conduites qui leur étaient habituelles. Les gestes, les attitudes, la disposition des acteurs, la topique des propos et le ton des discours, tout ce qui en fin de compte définissait les rôles et les figures des uns et des autres, appartenait davantage au système de représentation de l'auteur qu'il n'était déterminé par les circonstances de l'événement. On peut donc utiliser sans trop de scrupules les descriptions laissées par les auteurs de la fin du premier siècle, du deuxième siècle et du début du troisième siècle, Appien, Dion Cassius, Frontin, Hérodien, Plutarque, Suétone et Tacite surtout, en postulant que ces rituels d'interaction devaient être marqués par une certaine continuité, et employer avec davantage de précautions, dans un souci de comparaison, les auteurs antérieurs comme César, Denys d'Halicarnasse, Flavius Josèphe, Salluste ou Tite-Live, et postérieurs comme Ammien Marcellin.

Ainsi défini, l'ensemble de la documentation permet alors de souligner un certain nombre de traits récurrents qui permettent de restituer assez bien ce que pouvait être une contio de soldats réunis par leur chef.

Les circonstances qui imposaient la tenue d'une telle assemblée étaient certes extérieures aux situations de communication elles-mêmes mais elles en commandaient la tenue et le déroulement. Elles déterminaient leur inscription dans la trame diachronique qui organisait les narrations et les représentations. Elles correspondaient en fait à la nécessité où se trouvait le commandement d'établir, de rétablir ou de renforcer l'adhésion des hommes et l'unité de la troupe, chaque fois qu'une modification importante était annoncée ou qu'une difficulté nouvelle se présentait.

\footnotetext{
${ }^{7}$ F. PINA POLO, Las contiones civiles y militares en Roma, Saragosse, 1989. Cf. aussi J.B.CAMPBELL, o.c.
} 
Ainsi, autant qu'on peut le noter, les situations qui entraînaient la réunion d'une contio tenaient d'abord à un changement important dans la composition de la troupe ou du commandement: lorsque deux unités se réunissaient par exemple ${ }^{8}$ et surtout lorsque le chef changeait. C'était le cas en particulier lors des très nombreux épisodes qui apparaissent chez les historiens, de transmission ou d'usurpation du pouvoir impérial au cours desquels les soldats étaient amenés à sanctionner la modification qui intervenait ${ }^{9}$. À cela s'ajoutaient les situations politiquement difficiles: de crise dynastique, quand l'empereur était menacé ${ }^{10}$, quand des négociations se préparaient dans une guerre civile ${ }^{11}$ ou d'une façon plus générale lorsque le chef devait justifier [215] son comportement ${ }^{12}$. Les soldats étaient alors des partenaires et de véritables interlocuteurs dont il convenait d'obtenir l'appui.

$\mathrm{Au}$ cours d'une campagne, les contiones se tenaient soit avant ce que l'on pourrait appeler une épreuve, soit après. On pourrait compter dans la première catégorie, l'entrée en campagne elle-même ${ }^{13}$, la bataille ${ }^{14}$, la traversée d'une $\operatorname{mer}^{15}$, d'un fleuve ou d'un désert ou toute autre situation difficile dont la perspective provoquait nécessairement l'incertitude ou l'inquiétude des troupes ${ }^{16}$. On pourrait y ajouter l'arrivée d'une nouvelle imprévue qui contraignait parfois à réunir l'assemblée ${ }^{17}$. Dans la seconde série d'exemples, deux cas pouvaient se présenter puisque les efforts avaient nécessairement abouti à l'échec ou au succès. Dans le cas d'une victoire ou d'une campagne réussie, la contio était l'occasion pour le chef de faire l'éloge des troupes et de récompenser les légats, tribuns, centurions et soldats

\footnotetext{
${ }^{8}$ APP., B.C., II, 55 (César).

9 e.g., SUET., Div. Claud., 10, 4 (Claude); Oth., 6 (Othon); TAC., Ann., XII, 69 (Néron) ; DIO CASS., LXI, 3, 1

(Néron); LXIV, 22, 2 (Mucien) ; LXVI, 26, 3 (Domitien).

${ }^{10}$ SUET., Div. Claud., 26, 2 (Claude); TAC., Ann., XI , 35, 4 (Claude) ; XV, 72 (Néron) ; cf. aussi, Hist., III, 9 ; IV, $24 ; 32$ (partisans de Vespasien).

${ }^{11}$ APP., B.C., V, 39 (L. Antonius).

${ }^{12}$ APP., B.C., I, 57 (Sylla).

${ }^{13}$ APP., B.C., II, 55 (César) ; DIO CASS., XL, 19, 1-2 (Crassus).

${ }^{14}$ APP., B.C., II, 73-74 (César) ; IV, 89-100 ; 117 (Brutus et Cassius) ; 119-120 (Antoine et Octave) ; TAC., Ann., II, 14 (Germanicus) ; DIO CASS., L, 15, 4 (Antoine) ; 23, 3 (Octave).

${ }^{15}$ DIO CASS.= ZONAR., IX, 12 (Scipion I'Africain) ; LX, 19, 2 (Narcisse).

${ }^{16}$ Cf. I 'éclipse de lune avant Pydna, DIO CASS. = ZONAR, IX, 23.; PLINE, N.H., II, 153.

${ }^{17}$ PLUT., Pomp., 41.
} 
qui le méritaient ${ }^{18}$. Les cas de défaite étaient plus rares et conduisaient à des sanctions ${ }^{19}$. Il faudrait enfin compléter cette liste des circonstances récurrentes par les mutineries et toutes les situations de crise qui imposaient des mesures de répression ${ }^{20}$.

La tenue d'une contio répondait donc à une nécessité. Les hommes la réclamaient parfois. D'autres étaient convoquées même si l'ennemi risquait d'être informé de leur déroulement et des propos qui y seraient tenus ${ }^{21}$. Elles répondaient toutes à un besoin de communication qui s'imposait lorsque les circonstances commandaient un resserrement des liens entre les parties. Elles ouvraient et sanctionnaient les périodes d'effort, de danger, de tension et de crise. Elles renforçaient, inauguraient ou refondaient les relations entre les hommes et leur chef. Elles étaient ainsi l'occasion d'un rituel où se redéfinissaient les rôles des différentes parties dans un échange réciproque de reconnaissance.

Cette définition apparaissait dans l'organisation même du rassemblement. Une contio se tenait au camp, plus rarement en campagne, mais toujours à l'écart du combat. Elle se distinguait ainsi de la hortatio qui correspondait au discours que le chef tenait en présence de l'ennemi à l'instant même où les troupes étaient engagées. Elle était parfois précédée d'une lustratio qui constituait l'armée ${ }^{22}$. En ce sens dans ce rituel qui conservait une définition civique, les hommes et leur chef retrouvaient leur statut de magistrat et de soldats-citoyens. [216]

La définition qu'en donnait Festus était explicite: contio significat conventum, non tamen alienum, quam eum qui a magistratu vel a sacerdote publico per praeconem convocatur $^{23}$. Et de fait les attributs qui rappelaient que le général était avant tout le détenteur d'un imperium, se trouvaient représentés. Le tribunal tout d'abord qui permettait certes à celui qui y parlait de se faire entendre mais qui avait surtout pour fonction de lui donner de la majesté et de l'autorité par la distance qu'il instituait avec son auditoire. Il était indispensable.

\footnotetext{
${ }^{18}$ L'exemple le plus caractéristique, correspondant sans doute à un épisode considéré comme fondateur est celui de PLUT., Cor., 10 ; cf. aussi TAC., Ann., II, 22 (Germanicus) ; Hist., II, 57; 59 (Vitellius) ; III, 32 (Antonius Primus).

${ }^{19}$ TAC., Hist., II, 47 (Othon) ; DIO CASS., LXIII, 13 (id.).

${ }^{20}$ APP., Iber., 35-36 (Scipion I'Africain) ; B.C., II, 47 (César) ; V, 128 (Octave) ; DIO CASS. = ZONAR ., IX, 10 (Scipion I'Africain) ; XLI, 26 (César) ; TAC., Ann., I, 25-26 (Drusus); 29 (Drusus) ; 34 ; 39 (Germanicus) ; Hist., I, 82-84 (préfets et Othon).

${ }^{21}$ SUET., Div. Aug., 27, 3 ; il pouvait alors s'agir d'une ruse de guerre qui permettait de tromper l'ennemi cf. DIO CASS., XL, 31 (Labienus); FRONT., Strat., I, 8, 5.

${ }^{22}$ APP., B.C., IV, 89 (Brutus et Cassius) ; TAC., Ann., XV, 26 (Corbulon).

${ }^{23}$ P. 34 L.
} 
Plutarque racontait par exemple que lorsque la nouvelle de la mort de Mithridate parvint aux soldats de Pompée, ceux-ci, exigeant de leur chef qu'il les réunît immédiatement, en élevèrent un en toute hâte en empilant des bâts de bêtes de somme ${ }^{24}$. Il participait en effet pleinement à la définition de l'imperium: y monter signifiait l'exercer, en descendre signifiait l'abandonner $^{25}$. Les appariteurs enfin étaient presents: les praecones qui convoquaient l'assemblée $^{26}$ et les licteurs qui signifiaient eux aussi par la nature même de leur personne que celui qu'ils accompagnaient, exerçait l'imperium ${ }^{27}$.

Les soldats dans ce rassemblement retrouvaient cette définition civique qu'à aucun moment de l'histoire de la fin de la République et des débuts de l'Empire, ils n'avaient sans doute perdue. Ce même terme de contio dont Festus indiquait qu'il ne pouvait être autre chose qu'un rassemblement opéré ès qualités par un magistrat, n'était pas employé par les auteurs latins pour d'autres réunions que celles de citoyens ou de soldats. De la même façon

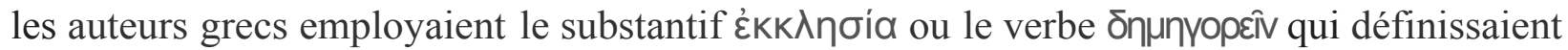
précisément une assemblée civique. Dans leur façon de se regrouper enfin les soldats conservaient leur statut. Ils ne se rassemblaient pas de façon désordonnée mais en prenant le poste qu'ils devaient occuper, à leur rang et sous leurs enseignes. Une des premières règles qu'observa en effet Germanicus en 14 quand il reprit en main les légions de Germanie, fut, avant même de s'adresser aux hommes, de leur ordonner de se répartir dans leurs unités ${ }^{28}$. Il fallait en effet que chaque individu fût à sa place pour que l'armée retrouvât sa définition collective.

Toutes ces indications convergent pour signifier que par la définition même des acteurs et la disposition qu'ils adoptaient, la contio correspondait à une rencontre qui associait le détenteur d'un imperium à ceux qui étaient soumis à son autorité et qui avait en même temps pour fonction de sanctionner le pouvoir de l'un et l'obéissance consentie des autres. Les deux aspects convergeaient en effet puisque l'armée et son chef ne tiraient leur existence que de leur relation réciproque. L'interaction des conduites de l'un et des autres n'avait alors d'autre effet que de définir le groupe qu'ils constituaient en commun par la manifestation simultanée du caractère propre des deux parties et de la solidité de leur entente. [217]

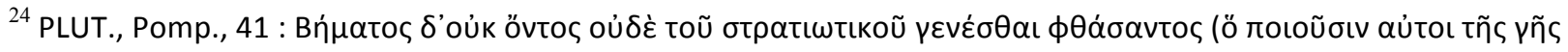

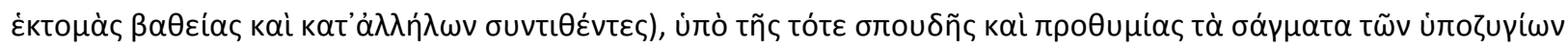

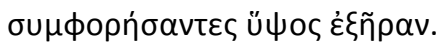

${ }^{25}$ APP., V, 128 (Octave) ; TAC., Ann., 1, 35 (Germanicus).

${ }^{26}$ APP., Iber., 35-36 (Scipion I'Africain) ; B.C., IV, 89-90 (Brutus et Cassius) ; DIO CASS. = ZONAR., IX, 12 (Scipion I 'Africain).

${ }^{27}$ Cf. B. GLADIGOW,Die sakrale Funktion der Liktoren, ANRW, 1, 2, 1973, pp. 295-313.

${ }^{28}$ TAC., Ann., I, 34 ; cf. aussi Hist., I, 82.
} 
Le chef jouait évidemment le rôle principal. Le style oratoire qu'il adoptait devait être conforme à son statut. Il exprimait l'autorité par la brièveté des propos - ce qu'indiquait Quintilien quand il soulignait que simpliciora militaris decent ${ }^{29}$, et la force de sa personnalité car, comme le notait Tacite à propos de Corbulon, c'était l'auctoritas quae pro viri militari pro facundia $\mathrm{erat}^{30}$.

La topique du discours qu'il tenait semble avoir été bien établie. Ses propos répondaient d'abord aux circonstances. À la veille d'une bataille par exemple, il décrivait la situation, préparait notamment les hommes à affronter les difficultés qui se présentaient. Il insistait sur leur force et la faiblesse de l'ennemi. Il les encourageait en expliquant parfois la tactique qu'il entendait adopter ${ }^{31}$. Il les inquiétait si nécessaire en décrivant les conséquences que la défaite entraînerait. Il promettait aussi les récompenses qu'ils attendaient ${ }^{32}$.

Souvent aussi, dans les moments difficiles, il évoquait et rappelait aux soldats le lien qui les lui associait, faisait appel au sacramentum ${ }^{33}$ les prenait à témoin de ses actes ${ }^{34}$ et réclamait leur fidélité. Il n'y avait pas de limite dans cette revendication de l'intensité de la relation. César fit même de ses hommes les témoins de son testament. Il se les associait ainsi comme amis et comme citoyens en rapprochant sans doute la procédure habituelle du testament secundum tabulas du souvenir de celle du testament in procinctu ${ }^{35}$. D'une façon générale, c'était la relation de fides qui engageait mutuellement le général et la troupe, qui était ainsi énoncée et mise en scène.

Le général n'était pas seul sur le tribunal. Il était sans doute habituellement entouré des membres de son consilium. Dans certains cas cependant, il s'adjoignait un certain nombre de personnages qui contribuaient à donner de l'autorité à ses propos ou qui leur apportaient le témoignage de leur présence ${ }^{36}$. Ce fut le cas par exemple de César qui, à la veille de franchir

\footnotetext{
${ }^{29} \mathrm{XI}$, I, 33 ; cf. TAC., Ann., XI , 35, 4 (Claude) ; Hist., I, 8 (Galba : imperatoria brevilate).

${ }^{30}$ Ann., XV, 26; cf. Hist., 111, 3.

31 TAC., Ann., II, 14 (Germanicus) ; DIO CASS., XL, I 9 (Crassus) ; 31 (Labienus) ; FRONT., Strat., I, 8, 5 ; II, 3 ; III, 1 , 1.

${ }^{32}$ SUET., Div. Claud., 10 ; Oth., 6 ; TAC., Ann., XII, 69 ; XV, 72, (Néron) ; Hist., I, 18 (Galba) ; II,82 (Mucien) ; HEROD., I, 6 (Commode).

${ }^{33}$ APP., B.C., II, 47 (César).

${ }^{34}$ TAC., Hist., III 9 ; IV, 24 ; 32.

${ }^{35}$ SUET., Div. IUl., 83.

${ }^{36}$ APP., B.C., 11, 50-51 (Pompée) ; IV, 89 (Brutus et Cassius) ; DIO CASS., XLVI, 56, 2 (triumvirs) ; le tribunal peut aussi servir de protection dans le cas d'une mutinerie (TAC., Ann., I, 39).
} 
le Rubicon, présenta à ses hommes les tribuns qui avaient fui Rome pour le rejoindre ${ }^{37}$ ou encore de Commode qui au moment de prendre le pouvoir se garantit de la présece des amis de son père ${ }^{38}$.

De leur côté, les soldats ne se comportaient pas en simples spectateurs. Ils intervenaient eux aussi par leurs cris ou pire, par leur silence. Les premiers manifestaient l'approbation. Les auteurs anciens insistaient en effet souvent sur l'enthousiasme avec lequel ils étaient poussés. Ils caractérisaient l'ardor ou l'ó $\rho \mu \eta^{39}$ de l'armée qui, si l'on en croit

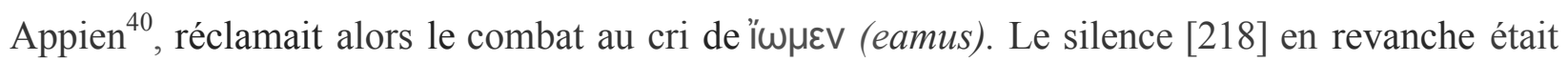
signe de démobilisation ou de désapprobation ${ }^{41}$. Quelquefois des protestations jaillissaient: elles annonçaient alors une véritable opposition qui était souvent signe de mutinerie ${ }^{42}$. En somme, si le rituel de la contio se déroulait comme il convenait, au discours du chef devait répondre un acquiescement des hommes qui révélait alors le consensus et manifestait l'adhésion.

La contio avait ainsi pour fonction de sanctionner la relation entre le général et ses soldats par la mise en place d'une reconnaissance réciproque. C'était particulièrement vrai dans le cas des assemblées qui marquaient la fin d'une campagne. Elles étaient en effet l'occasion pour le chef de faire l'éloge des hommes par une laudatio et de récompenser ceux qui l'avaient mérité par l'attribution de dona militaria ou même, dans quelques cas attestés à la fin de la République, par une élévation dans le statut civique par la concession de la citoyenneté ou de l'anneau d'or ${ }^{43}$. Ces décisions avaient valeur juridique. Certains bénéficiaires les faisaient mentionner parmi leurs titres ${ }^{44}$. Parfois surtout, la mention d'une décision annoncée in contione signifiait qu'il s'agissait d'un acte officiel qui avait la même portée qu'un édit ou un rescrit ${ }^{45}$. La récompense ou la promotion reconnaissait ainsi le statut civique et militaire des soldats et le sanctionnait.

\footnotetext{
${ }^{37}$ APP., B. C., II , 33 ; DIO CASS., XLI, 4, 1; SUET., Div. IUl., 33.

${ }^{38}$ HEROD., I. 5.

${ }^{39}$ APP., B.C., II, 52 (Pompée); 54 (César); III, 86-87 (Octave); TAC., Ann., II, 14-15 (Germanicus).

${ }^{40}$ B.C., IV, 94.

${ }^{41}$ APP., B.C., III, 43 (Antoine) ; TAC., Hist., I, 18 (Galba et Pison).

42 TAC., Ann., I, 25-26; 29 (Drusus) ; 34 (Germanicus).

${ }^{43}$ CIC., ProArch., 24 (Pompée) ; VAL. MAX., VIII, 4, 3 ; CIC., 2 Verr : III, 185.

${ }^{44}$ Cf. CIL, II, 2079; VI, 3617; A.E., 1956, 124.

${ }^{45}$ Cf. Fgt. Vatic., 195.
} 
Les jugements négatifs et les punitions qui étaient énoncés et ordonnés au cours d'une contio, avaient le même effet. Ils aboutissaient à la mise à l'écart ou à l'exécution de ceux qui avaient démérité ou désobéi. En ce sens, ils éliminaient les mauvais pour conserver les bons. L'armée était alors régénérée et reconstituée dans sa définition première.

En réponse également, les cris des soldats reconnaissaient et sanctionnaient l'autorité du chef. C'était le cas bien évidemment lors des salutations impériales qui marquaient les débuts de règne ou les victoires ${ }^{46}$. Ces manifestations étaient nécessaires à la définition même de limperium. Aussi bien redoutait-on plus que tout le silence ou même la mollesse de certaines réponses qui ne permettaient pas de fonder un pouvoir solide ${ }^{47}$. Un épisode un peu particulier permet également de bien souligner le phénomène. Lorsqu'en 43, - racontait Dion Cassius les troupes de Claude hésitèrent un instant au moment de traverser la Manche, Narcisse s'adressa à elles pour les encourager. La réponse vint sous la forme du cri "Io Saturnalia" qui rappelait à l'ami de Claude sa condition d'affranchi ${ }^{48}$. Il s'agissait bien d'une acclamation mais qui discréditait et rejetait celui qui en faisait l'objet. Les soldats reproduisaient dans ce cas la pratique habituelle, en conservaient la fonction mais la tournaient contre celui qui osait leur parler sans en avoir le statut. Ils ne pouvaient en effet reconnaître l'autorité d'un tel chef sans se disqualifier eux-mêmes.

Plus durement encore la contio pouvait s'achever sur un échec de celui qui l'avait convoquée. L'abdication était au bout; au moins simulée comme dans l'épisode de la mutinerie de 14 où Germanicus, incapable de maîtriser les manifestations des soldats, [219] finit par abandonner le tribunal et par menacer de se suicider avant de pouvoir, mais un peu plus tard, reprendre la situation en main ${ }^{49}$.

La contio qui réunissait le chef et ses hommes constituait donc un rituel politique extrêmement important. L'échange qui s'établissait alors avait fondamentalement pour fonction de réaffirmer le statut des uns et des autres, de le refonder en quelque sorte dans une reconnaissance réciproque qui marquait l'adhésion et le consensus.

Cette économie d'ensemble du rituel que constituait la contio qui apparaît ainsi à travers des traits récurrents qui se dégagent de la lecture des sources littéraires, n'a évidemment jamais été donnée comme telle de façon explicite. Les historiens étaient en effet trop soumis aux contraintes de la narration pour en avoir à aucun moment fourni une description systématique. Aucun d'entre eux, par exemple, n'a jamais envisagé de décrire ou de

\footnotetext{
${ }^{46}$ E.g. TAC., Ann., XII, 69, 4 (Néron).

${ }^{47}$ TAC., Hist., I, 18 (Galba et Pison) ; HEROD. , II, 2, 5-8 (Pertinax).

${ }^{48}$ DIO CASS., LX, 19, 2.

${ }^{49}$ TAC., Arm., I, 35, 4.
} 
raconter toutes les contiones qui se seraient déroulées au cours des événements qu'il évoquait alors qu'on peut être assuré qu'il s'en réunissait au moment de chaque bataille ou prise de commandement. C'était en effet en fonction de la stratégie générale de narration qu'ils avaient adoptée que les auteurs antiques choisissaient ou non d'évoquer ces épisodes. Étaient donc retenues les contiones qui constituaient un événement important par elles-mêmes : prise de pouvoir, sanction d'une mutinerie, ou qui permettaient d'inscrire un discours dans la narration ou encore qui servaient d'introduction à une bataille décisive. Dans la plupart des cas cependant, les informations dont l'historien disposait étaient tout à la fois insuffisantes et trop diverses. Il était amené en effet à reconstituer à partir de son expérience et de son imagination une foule de données qui ne lui étaient pas livrées par ses sources; ce qui lui permettait également de témoigner de son habileté et de sa compétence, notamment lorsqu'il recomposait des discours qui y auraient été tenus. Il était aussi amené à laisser de côté tout ce qui n'avait aucun intérêt dans la perspective où il se plaçait et donc à n'évoquer que les détails qui contribuaient à la dramatisation de l'épisode.

C'est ainsi que toutes les narrations devaient nécessairement indiquer les circonstances qui conduisaient à la tenue d'une contio puisqu'elles étaient inscrites dans le déroulement même de l'action. Elles devaient aussi désigner les chefs qui les tenaient puisqu'ils étaient les acteurs de l'épisode. Mais l'évocation des autres traits dépendait en revanche de leur pertinence avec le sens général de l'événement et de l'intensité évocatoire qu'ils étaient susceptibles de lui apporter. Tous n'étaient donc pas indiqués.

Les attributs qui soulignaient la possession de l'imperium dans la personne du chef n'étaient évoqués que lorsqu'il convenait d'insister sur un aspect particulier de son statut ou que leur présence permettait de dramatiser la situation. C'est ainsi qu'Appien, en évoquant les praecones qui convoquaient la contio ordonnée par Scipion l'Africain pour réprimer une mutinerie, donnait à l'événement une intensité qui en préparait le dénouement tragique ${ }^{50}$. Dans d'autres cas en revanche, l'évocation du tribunal servait à souligner le caractère hâtif ou improvisé du rassemblement par l'emploi d'une estrade de fortune comme dans ce cas que l'on a déjà cité de l'annonce de la mort de Mithridate à Pompée ${ }^{51}$, ou encore la tension et l'urgence de situations qui contraignaient un empereur à s'adresser à ses hommes du haut d'un vallum ou des [220] marches d'un palais ${ }^{52}$.

De la même façon, les historiens ne décrivaient guère le chef que lorsqu'ils souhaitaient faire apparaître ses qualités et son autorité ou au contraire ses faiblesses : la force et l'autorité de

\footnotetext{
${ }^{50}$ APP., lber : 35 .

${ }^{51}$ PLUT., Pomp., 41, 4-5.

52 TAC., Hist., 1, 29 (Pison) ; I, 36 (Othon) ; HEROD., 11, 6 (Didius Julianus) ; cf. aussi DIO CASS., LIX, 17 (Caligula).
} 
Septime Sévère ${ }^{53}$, les gestes pathétiques de César déchirant ses vêtements ${ }^{54}$ ou encore la jeunesse de Néron lisant un discours rédigé par Sénèque ${ }^{55}$. Les personnages qui l'entouraient n'étaient évoqués que lorsqu'effectivement leur présence avait une fonction précise dans le récit et soulignait le besoin qu'éprouvait le général de les faire apparaître à ses côtés. C'était en effet la difficulté de la situation où se trouvait ce personnage ou au contraire la position d'autorité qu'il avait gagnée, qui conduisait l'auteur de la narration à attirer l'attention du lecteur sur ce point.

La présentation de la troupe obéissait aux mêmes nécessités. Il fallait le plus souvent des circonstances dramatiques pour qu'elle apparût dans le récit. Une situation de consensus n'avait guère d'intérêt en effet à moins que ne s'y révélât un enthousiasme particulier de la part des soldats ${ }^{56}$. Les mouvements d'indiscipline, en revanche, étaient autrement plus intéressants en ce qu'ils étaient l'occasion de scènes plus intenses. C'est ainsi que Tacite pouvait se permettre de décrire la grande manifestation pathétique des mutins de 14 qui dénudaient leurs corps et exhibaient leurs cicatrices en réclamant leur démobilisation ${ }^{57}$. Il pouvait aussi, à propos de la présentation de Pison par Galba, souligner le manque d'enthousiasme de l'armée en mettant en évidence le contraste entre les tribuns et les centurions qui aux premiers rangs réagissaient favorablement, et les soldats du fond de l'assemblée qui eux demeuraient silencieux ${ }^{58}$. Parfois enfin, la contio pouvait être l'occasion de malentendus comme cet épisode évoqué par Suétone au cours duquel les soldats, qui étaient trop loin de César pour pouvoir l'entendre, crurent que le geste par lequel il montrait son anneau d'or, indiquait qu'il leur promettait l'accès à l'ordre équestre ${ }^{59}$.

Le but des historiens n'était donc pas de présenter à chaque fois une description générale et complète de la contio. Bien au contraire, les aspects les plus particuliers ou les plus dramatiques étaient ceux qui retenaient l'attention. Sa nécessité profonde qui tenait à ce qu'elle était l'occasion d'une refondation des rapports entre le chef et ses hommes et de la réaffirmation du consensus, n'était nullement contestée, mais c'étaient les épisodes les plus éclatants ou au contraire les plus difficiles du processus dont elle était l'occasion qui étaient mis en valeur.

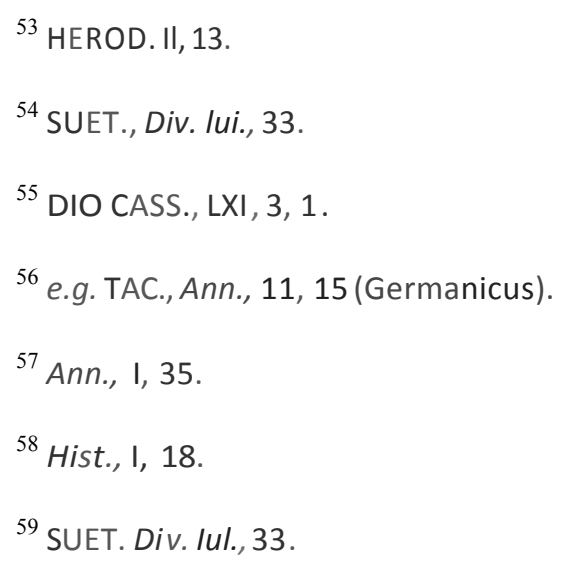


Les représentations de contiones sur les colonnes de Trajan et de Marc Aurèle devaient en revanche répondre à bien d'autres nécessités qui tenaient soit aux contraintes qu'imposait la construction de l'image elle-même, soit au projet d'ensemble qui était la célébration de la gloire impériale ${ }^{60}$. [221]

Les premières tenaient à ce que l'image offrait un champ de présentation limité mais où tout ce qui y était indiqué devait l'être de façon complète. C'est ainsi que des détails que les historiens n'indiquaient que de façon épisodique étaient nécessairement présents : le tribunal ou la tenue physique et vestimentaire des participants étaient systématiquement dépeints. D'autres en revanche étaient par force symbolisés. Seul un petit nombre de soldats par exemple pouvait être représenté. Les gestes ne pouvaient être que suggérés. Les discours enfin n'avaient aucune place sinon par la topique que sous-entendait le contexte dans lequel la scène se trouvait insérée.

La célébration de la gloire impériale imposait évidemment de représenter l'empereur et interdisait en revanche que des manifestations de désaccord ou d'indiscipline pussent apparaître. La représentation de la contio n'avait pour fonction que de manifester le consensus accompli et efficace qui régnait entre le chef et ses hommes. Toutes les indications iconographiques devaient donc y conduire. L'image euphémisait donc, à la différence de la narration qui, elle, dramatisait. Elle contraignait en conséquence à d'autres choix que ceux auxquels se soumettaient les historiens.

Les circonstances de la contio qui, dans la narration, étaient nécessairement présentes puisque c'étaient elles qui commandaient qu'elle fût évoquée, n'avaient plus du tout le même intérêt. La nécessité de la représentation iconographique tenait simplement à ce qu'elle était un des rites nécessaires à l'exercice de l'imperium. La fonction de la colonne imposait donc que des contiones y fussent représentées mais de façon convenue aux moments les plus attendus.

Les mêmes difficultés qui commandaient l'organisation d'ensemble de l'iconographie de la colonne s'imposaient alors. Puisque, comme on l'a souvent répété, la lecture des scènes ne pouvait être syntagmatique que dans les parties basses et que c'était notamment une lecture paradigmatique qui s'imposait dans les parties hautes, les relations diachroniques ne pouvaient apparaître clairement que dans les premières spires. C'est ainsi que sur les deux colonnes, les contiones apparaissaient dans la partie inférieure en début et en fin de campagne ${ }^{61}$ et d'une façon générale avant ou après des batailles et des moments d'efforts ${ }^{62}$; ce qui, on l'a vu, correspondait à leur fonction normale qui était de permettre d'encourager les hommes avant un

\footnotetext{
${ }^{60}$ Cf. d 'une façon générale, N. HANNESTAD, Roman art and Imperial Policy, Aarhus, 1988, pp. 164-165 ; $241-242$.

${ }^{61} \mathrm{CT}, 10 ; 54 ; 77 ; 104 ; \mathrm{CA}, 4$ [fig. 14] ; 55 [fig. 75-76].

${ }^{62}$ CT, $27 ; 42 ; 73 ; 125 ; 137$; CA, 9 [fig. 23-24] ; 83 [fig. 105] ; 96 [fig. 111-112] ; 100 [fig. 120].
} 
moment difficile ou périlleux et de les récompenser une fois le succès obtenu. Plus haut, en revanche, les liens avec la narration d'ensemble étaient beaucoup moins compréhensibles. 11 n'est pas toujours possible par exemple de déterminer si la scène de contio devait être associée avec l'épisode qui suivait ou qui précédait ${ }^{63}$. Les relations diachroniques étaient alors sans doute relayées par des relations synchroniques ou en tout cas non séquentielles qui donnaient aux scènes de contiones leur efficacité en en reprenant d'autres du même type ou en les opposant aux scènes de marche, d'exercices ou de combat qui étaient placées au dessus ou au dessous d'elles $^{64}$. Elles retrouvaient alors dans cette contiguïté verticale la fonction qui faisait [222] d'elles ces moments de refondation de l'armée qui délimitaient et scandaient les phases d'effort. Elles échappaient ainsi à la narration continue des événements pour prendre la forme de manifestations paradigmatiques de l'adhésion de l'armée à son chef qui se renouvelaient régulièrement tout au long de la colonne de la même façon que leur répétition au cours des campagnes avait été indispensable à la bonne conduite des opérations. Elles jouaient ainsi le même rôle que ces scènes de consilium ou de réception d'ambassades qui réaffirmaient par leur récurrence l'intelligence ou la clémence de l'empereur. Dans leur structure générale, les représentations des contiones respectaient les nécessités profondes de la mise en œuvre de l'interaction et de l'affirmation du consensus qui correspondaient à leur définition.

L'autorité de l'empereur était ainsi particulièrement soulignée. Chaque scène le présentait debout sur un tribunal, bien identifiable, la main droite levée ; ce qui signifiait qu'il était en train de tenir un discours ${ }^{65}$. S'adresser aux hommes était indispensable à la mise en œuvre de l'interaction et à la définition même de son pouvoir. Le geste était donc bien marqué et clairement visible jusque sur les spires les plus hautes. Un groupe l'entourait, qui représentait son consilium et ceux qu'éventuellement il s'était adjoints, et qui, comme on l'a vu, contribuaient à la définition de son imperium et de son autorité ${ }^{66}$. D'autres traits caractéristiques du pouvoir pouvaient éventuellement être ajoutés : la hasta que sur certaines

\footnotetext{
${ }^{63}$ En ce sens le classement opéré pour la colonne Trajane par L. E. BAUMER, J. d.I., 106, 1991, pp. 278287, entre "Anfangs-Adlocutiones", "Sieges-Adlocutiones" et "Schluß-Adlocutiones" déjà un peu fragile pour la partie haute de la colonne Trajane dans la mesure où le statut de la scène 137 n'apparaît pas clairement, ne semble pas pertinent pour la partie haute de la colonne Aurélienne.

${ }^{64}$ Cf. pour la colonne Trajane, les analyses de S. SETTIS dans id., ed., La Colonna Traiana, Turin, 1988, pp. 164-187.

${ }^{65}$ Le geste signifie fondamentalement la tenue du discours même si par ailleurs il joue un rôle dans la composition de l'image (cf. R. BRILLIANT, Gesture and Rank in Roman Art, Mem. Connecticut Acad. Art Sc., 14, 1963, New Haven, 1963 ; S. SETTIS, o.c., pp. 151-152).

${ }^{66}$ Les différences stylistiques entre la colonne Trajane et la colonne Aurélienne notées par G. BECATTI, La colonna coclide istoriata, Problemi storici iconografici stilistici, Rome, 1960, p. 74, ne contredisent pas cette disposition d'ensemble.
} 
scènes, il tenait ${ }^{67}$, ou encore les licteurs qui parfois l'accompagnaient ${ }^{68}$. D'autres nuances intervenaient encore : la toge et le manteau à la place de la cuirasse ${ }^{69}$, la lecture d'un texte à la place de la tenue d'un discours ${ }^{70}$, dont la signification convergeait avec la définition d'ensemble mais en ajoutant des particularités dont le sens parfois nous échappe ${ }^{71}$.

La façon dont les soldats étaient représentés, répondait évidemment au rôle qu'ils étaient susceptibles de jouer dans ces contiones. Les enseignes de leurs unités étaient toujours figurées : elles signifiaient que c'était par corps qu'ils étaient rassemblés, chacun à son rang et à sa place, et non sous la forme d'une foule confuse qui aurait signifié le désordre et l'indiscipline. Leur adhésion et leur obéissance ne faisait aucun doute. Elle était leur réponse au discours de leur chef, la part qu'ils prenaient dans cette interaction qui fondait le consensus. Le cercle qu'en contradiction parfois avec la disposition manipulaire, ils formaient généralement autour du tribunal manifestait leur [223] empressement. Les têtes toujours levées et les regards tendus vers leurs chefs révélaient leur attachement. Cette disposition d'ensemble était elle aussi bien visible. Elle était le signe iconographique du succès de la contio. Leur groupe cependant connaissait lui aussi certaines variations dans la présentation. Dans la plupart des cas, les soldats étaient en armes, parfois au contraire, ils étaient désarmés ${ }^{72}$ Certains d'entre eux parfois se distinguaient: ils regardaient dans une autre direction ${ }^{73}$. D'autres surtout levaient la main droite ${ }^{74}$; ce qui signifiait là aussi qu'ils parlaient et donc, puisque, dans le contexte idéologique des colonnes, toute manifestation d'opposition était inenvisageable, qu'ils manifestaient leur adhésion et acclamaient l'empereur.

${ }^{67}$ CT, 27; CA, 96 [fig. 111-112] ; 100 [fig. 120].

${ }^{68} \mathrm{CT}, 104 ;$ CA, 4 [fig. 14] ; 55 [fig. 75-76].

${ }^{69}$ CT, 77; CA, 4 [fig. 14] ; 9 [fig. 23] ; 55 [fig. 75-76] ; 83 [fig. 105] ; 100 [Fig. 120].

${ }^{70}$ CA, 9 [fig. 23] ; 55 [fig. 75-76]. D'une façon générale, ces variations peuvent avoir eu de multiples significations qui nous échappent pour la plupart. II ne s'agit cependant certainement pas de représentations de la réalité ou de caractérisations de l'individu (cf. K. LEHMANN- HARTLEBEN, Die Trajanssäule, p. 15).

${ }^{71}$ Les allusions à la lecture de documents que l'on trouve chez les historiens correspondent à des chefs rapportant à leurs hommes les indications d'autrui (TAC., Ann., I, 25-26; Hist., III, 3 ; 9 ; IV, 24 ; 32). On ne peut donc pas les rapprocher de ces représentations où c'est l'empereur lui même, source de toute autorité, qui lit.

${ }^{72}$ CT, 77 ; CA, 83 [fig. 105] ; 100 [fig. 120].

${ }^{73} \mathrm{CT}, 104 ; 125$; CA, 100 [fig. 120].

${ }^{74} \mathrm{CT}, 77 ; 104 ; 125 ; 137$; CA, 55 [fig. 75] ; 83 [fig. 105]. Contrairement à ce que pensent certains auteurs (cf. en part., C. CICHORIUS, Die Reliefs der Traianssäule, III, Berlin, 1900, pp.281-284 ;K. LEHMANN-HARTLEBEN, Die Trajanssäule, p. 12), la preuve n'est pas faite qu'il s'agisse précisément de salutations impériales qui n'apparaissent pas dans les descriptions que les historiens ont données de contiones. 
Toutes les scènes enfin n'étaient pas traitées de la même façon. Certaines, dans le cours de la présentation, n'occupaient qu'une place relativement peu importante. D'autres en revanche semblent avoir compté davantage. Celle notamment qui clôturait la première campagne de la colonne de Marc Aurèle ${ }^{75}$ frappe par son ampleur: des licteurs sont présents, l'empereur y lit un texte, deux personnages sur le côté gauche semblent manifester de façon plus vive leur adhésion. À ce point du déroulement de la narration, il s'agissait évidemment de cette contio de fin de campagne qui était l'occasion d'une laudatio et de récompenses. Sans doute était-ce sur ce point que l'auteur voulait insister en en marquant le caractère officiel par la présence des licteurs et en isolant deux individus dans lesquels on pourrait bien reconnaître des bénéficiaires de la reconnaissance impériale.

Ainsi se trouvaient respectées dans les représentations de deux colonnes les nécessités sémantiques profondes qui étaient à l'œuvre dans les contiones. La structure d'ensemble de la scène présentait en effet l'empereur muni de tous les attributs de l'imperium et son armée définie comme telle par la tenue des soldats et l'ordre de leurs enseignes. Elle associait les deux parties dans un rituel d'interaction où les gestes de l'un et la convergence des attitudes des autres manifestaient par la cohérence du groupe et l'adhésion des hommes, la légitimité et

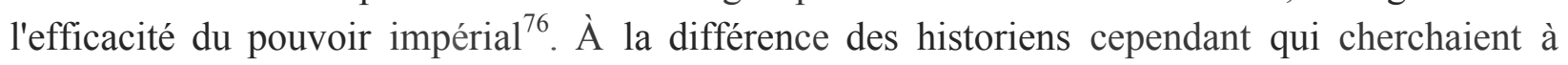
souligner la supériorité des grands chefs ou l'incompétence des tyrans et qui y parvenaient par la dramatisation et l'insistance sur certains détails importants, les auteurs des scènes représentées sur les colonnes étaient soumis à une double contrainte. La première tenait à ce qu'ils n'avaient d'autre choix que de signifier la profondeur de l'accord qui liait les soldats à l'empereur et fondait la solidité de son pouvoir. La seconde était celle de l'instrument même de communication qu'ils utilisaient. Les seuls signes qui pouvaient manifester l'entente entre les partenaires reposaient sur la disposition des personnages, les gestes et les attitudes qu'ils pouvaient [224] leur prêter. Ils constituaient donc des invariants de la représentation.

Mais on comprend aussi l'importance de ces variations qui d'une scène à l'autre permettaient d'éviter la monotonie tout en conservant l'essentiel. Des gestes légèrement différents, des costumes qui changeaient çà et là sans altérer le statut de ceux qui les portaient $^{77}$, des détails divers mais qui appartenaient toujours au même registre symbolique,

\footnotetext{
${ }^{75}$ CA, 55 [fig. 75-76].

${ }^{76}$ En ce sens, il est difficile de privilégier un trait plutôt qu'un autre comme la vertu impériale (S. SETTIS, Annales, E.S.C., 1985, pp. 1162-1163 ou la fides exercitus (L. E. BAUMER, J.d.I., 106, 1991, p. 285) qui constituent une partie de l'ensemble énoncé.

${ }^{77}$ Les historiens de l'art ont le plus souvent considéré que les différences de cuirasse entre les soldats permettaient de distinguer entre les légionnaires et les auxiliaires. À aucun moment, les narrations des historiens ne font apparaître une telle distinction. L'armée y est considérée comme un tout ou quand elle est partagée en plusieurs unités auxquelles le chef s'adresse séparément, c'est pour des raisons tactiques (DIO CASS., LXII, 9-11, cet épisode correspond en fait à une hortatio). Une telle affirmation repose en fait sur le
} 
étaient d'autant plus indispensables que la rencontre des nécessités idéologiques et iconographiques imposait un schéma répétitif. Et d'ailleurs, cette règle qui apparaît si nettement à propos des contiones devrait sans doute être élargie à l'ensemble des représentations puisque ces mêmes contraintes s'exerçaient sur chaque type de scène. Elle est ainsi une des principales explications de ces différences de style plastique et de choix des objets présentés qui sautent aux yeux lorsque l'on compare les deux colonnes. Elle signifie que la manifestation du pouvoir impérial n'avait rien de figé. L’inventivité de la poétique sculpturale révélait la capacité toujours vivante du pouvoir impérial à provoquer la reconnaissance par le peuple romain de sa supériorité et de son efficacité en renouvelant les sources de son admiration. L'empereur de la colonne Aurélienne ne reproduisait pas celui de la colonne Trajane : il le prolongeait et l'exhaussait en amplifiant les manifestations de sa gloire.

L'intérêt des scènes de contio qui apparaissent sur les colonnes Trajane et Aurélienne tient ainsi à ce qu'il permet de réaffirmer l'extrême importance du rôle que ces rassemblements jouaient dans la définition du pouvoir impérial. C'était là en effet que se mettait en place, dans le rituel de l'interaction des paroles et des gestes, la reconnaissance réciproque qui légitimait et renforçait l'autorité du Prince et assurait le consentement et l'obéissance des soldats - et toujours citoyens - de l'Empire. Leur répétition à distances régulières le long des fûts le confirme assurément, qui garantissait aux yeux du spectateur cette cohésion qui avait permis la victoire. Elle ne se contentait donc pas d'afficher la vertu impériale. Elle mettait en scène tous les partenaires qui contribuaient par leur attachement et l'énergie qu'ils mettaient à défendre l'Empire, la légitimité et la force d'une monarchie indissociable de son exercice guerrier. En ce sens, elles ne forçaient pas simplement l'admiration, elles élargissaient symboliquement à tous les citoyens les responsabilités et les succès des combats à mener contre les Barbares pour le maintien de la suprématie et de la paix.

Ainsi se confirme le lien profond et continu qui unissait les conduites politiques et leur reprèsentation. C'était la même nécessité de fonder la cohésion qui contraignait les chefs à réunir leurs hommes, à s'adresser à eux, à obtenir leur acquiescement et à sanctionner les succès ou les échecs, qui imposait la figuration de ces scènes sur les monuments à la gloire de la monarchie impériale. Là, soumise à l'évidence de la victoire, à l'abri des incertitudes et des crises dont les historiens se faisaient parfois l'écho, se manifestait de façon éclatante et vivante cette articulation du pouvoir et de l'adhésion qui fondait la légitimité de l'Empire et contraignait le spectateur à prendre sa part du consensus. [225]

Note à propos de la scène VI (fig. 17) de la colonne Aurélienne

postulat d'une représentation réaliste et anecdotique (cf. en part. C. CICHORIUS, Die Reliefs der Traianssäule, ad loc. ). Il vaut donc mieux être prudent et considérer que ces différences relèvent de la variation. 
Le relief représentant cette scène est extrêmement dégradé et toute identification ne peut être que totalement hypothétique. Celle qui est faite depuis Petersen ${ }^{78}$ avec une lustratio par comparaison avec la scène 8 de la colonne Trajane ne correspond pas aux indications que donnent les sources littéraires. À chaque fois en effet que l'une d'entre elles fait allusion à la mise en ouvre d'une lustratio, celle-ci est accomplie avant la tenue de la contio ${ }^{79}$. Cette séquence narrative correspond parfaitement à la situation de la colonne Trajane où les lustrationes des scènes 8,53 et 103 précèdent les contiones des scènes 10,54 et $104^{80}$. Mais elle ne peut être envisagée pour la colonne Aurélienne puisque la contio qui devrait correspondre à la scène VI, serait celle de la scène IV (fig. 14-15) qui la précède. [226]

Jean-Michel DAVID

Université Paris 1 Panthéon-Sorbonne

UMR 8210 ANHIMA

\footnotetext{
${ }^{78}$ Cf. E. PETEREN, A. VON DOMASZEWSKI, G. CALDERINI, Die Marcus-Säule auf Piazza Colonna in Rom, Munich,1896, p. 54.

${ }^{79}$ Cf. notamment TAC., Ann., XV, 26, 2 ; APP., B.C., IV, 89 ; DIO CASS., LXI V, 16.

${ }^{80}$ Cf. L. WINKLER dans J.d.I., 106, 1991, pp. 268-271.
} 